showed increased inhibitory and excitatory synaptic connectivity between postnatal day P10 and P18, rats exposed at P7 to a single dose of phenobarbital, phenytoin, or lamotrigine had impaired maturation of synaptic connectivity. Phenobarbital exposure also impaired striatal-mediated behavior on P25. Neuroprotective pretreatment with melatonin, which prevents drug-induced neurodevelopmental apoptosis, prevented the drug-induced disruption in maturation. Synaptic development was not disrupted by levetiracetam. (Forcelli PA, Janssen MJ, Vicini S, Gale K. Neonatal exposure to antiepileptic drugs disrupts striatal synaptic development. Ann Neurol 2012 Sep;72(3):363-72). (Respond: Dr Forcelli or Dr Gale, Department of Pharmacology and Physiology, Georgetown University, Washington, DC. E-mail: Paf22@georgetown.edu).

COMMENT. Protection from experimental seizures in small, laboratory animals is the backbone of development of new potentially effective and less toxic antiepileptic drugs, beginning with the introduction of phenytoin in 1937 (Putnam TJ, Merritt HH. Science 1937 May 28;85(2213):525-6). Phenobarbital, introduced as an anticonvulsant by Hauptmann in 1912, has a troublesome sedative side effect. Phenytoin was the first AED to show anticonvulsant activity without sedation. Various laboratory methods to elicit seizures have been employed in the testing of new drugs, and major advances were made in the mid 1900s, particularly in the pharmacology department at the University of Utah, under the direction of Louis S Goodman, Ewart A Swinyard, Dixon M Woodbury and others. The current laboratory study from Georgetown University should increase concern regarding the potential hazard of continuing use of phenobarbital and phenytoin in the neonate and young infant, and should lead to the introduction of alternative therapies that control seizures without compromising synaptic maturation, cognition and behavior.

Epileptologists in a Catch-22 situation. Whereas this pharmacological study cautions the epileptologist against overuse of AEDs in the control of neonatal seizures, the following clinical study emphasizes the need for early aggressive treatment and seizure control in infants and young children.

\title{
AGE AT ONSET OF EPILEPSY AND EFFECT OF UNCONTROLLED SEIZURES ON COGNITIVE OUTCOME
}

Researchers at the Epilepsy Center and Department of Psychiatry at Lurie Children's Hospital of Chicago examined the association of cognitive scores and age at onset of epilepsy, pharmacoresistance, and interaction between the two in a prospective community-based study of 198 children, aged $<8$ years, with new-onset epilepsy. The range of epilepsy syndromes reflected those seen in this age group. The average age at the first unprovoked seizure was 3.7 years. Full-scale IQ (FSIQ) assessed with the WISC for Children (WISC-III) after 8-9 years follow-up (mean IQ 94.2) was not correlated with age at onset. In $38(19.2 \%)$ patients meeting the study criteria, pharmacoresistance was associated with an 11.4-point lower FSIQ $(\mathrm{p}=0.002)$ and similar decrements in each WISC-III domain. Pharmacoresistance lessened with increasing age. IQ was strongly correlated with age at onset in the pharmacoresistant group $(\mathrm{p}<0.0001)$ but not in the nonpharmacoresistant group $(p=0.61)$. Impairment of cognitive function associated with uncontrolled seizures is most severe in infancy and lessens with increasing age at onset. 
The findings emphasize the need for early aggressive treatment and control of seizures in infants and young children. (Berg AT, Zelko FA, Levy SR, Testa FM. Age at onset of epilepsy, pharmacoresistance, and cognitive outcomes. A prospective cohort study. Neurology 2012 Sep 25;79(13):1384-91).

COMMENT. This study corroborates the clinical and laboratory evidence pointing to an injurious effect of seizures and epileptic encephalopathy on cognition, especially in the young. In a previous analysis of very young patients in this cohort (seizure onset 0-3 years), Vineland Adaptive Behavior Scale (VABS) scores declined over the first 3 years in patients with pharmacoresistant seizures but stayed constant in those with well-controlled seizures (Berg AT et al. Pediatrics 2004 Sep;114(3):645-50). The effect of seizures on the developing brain appears to be potentially more injurious than that of sedative anticonvulsants.

\section{VASCULAR DISORDERS}

\section{CYTOKINE MARKERS OF FOCAL CEREBRAL ARTERIOPATHY}

Researchers at the University of Calgary, Canada report 2 adolescents with focal cerebral arteriopathy (FCA), a stenosis of the large or medium-sized blood vessels, with marked elevations in inflammatory biomarkers. These compared to a case of nonarteriopathic arterial ischemic stroke (AIS) and normal adult serum. Case 1. A 14year-old girl presented with acute left hemiparesis secondary to right MCA AIS. Vascular imaging revealed arteriopathy with irregular stenosis and dilatation of internal carotid, M1, M2, and A1 arteries without dissection. She recovered following anticoagulates and treatment with steroids and cyclophosphamide. Case 2. A healthy 15-year-old boy presented with aphasia and hemiparesis secondary to large left MCA AIS. A malignant cerebral edema required decompressive hemicraniectomy. Following treatment with aspirin and steroids the arteriopathy partially improved over months. Case 3. A 16-yearold female smoker after starting on contraceptives presented with right hemiplegia and left M1 thrombus without arteriopathy. Echocardiogram revealed a patent foramen ovale, and an embolism was presumed. At 6 months she is normal and taking aspirin.

Cytokine assays were quantified for each subject. Elevations of inflammatory markers were seen in acute phase of FCA cases but not in the embolic case. These elevations normalized following treatment. Quantitative analysis of serum cytokine is a useful biomarker in FCA and childhood stroke. (Mineyko A, Narendran A, Fritzler ML, Wei X-C, Schmeling H, Kirton A. Inflammatory biomarkers of pediatric focal cerebral arteriopathy. Neurology 2012 Sep 25;79(13):1406-8). (Response: Dr Mineyko, E-mail: Aleksandra.mineyko@alberthealthservices.ca).

COMMENT. An editorial comment questions whether the improvement in treated patients could be part of the natural history. In a recent series of 79 children with stroke due to FCA, $23 \%$ normalized, $77 \%$ stabilized or improved, and only $6 \%$ showed progression (Braun KP et al. Brain 2009 Feb;132(2):544-57). Studies in large cohorts are required to clarify the utility of measuring inflammatory markers in children with stroke. 